\section{Absorbance accuracy check on the LKB 8600 reaction rate analyser}

\author{
D. F. DAVIDSON Department of Biochemistry, Gart- \\ navel General Hospital, Glasgow
}

The LKB 8600 reaction rate analyser measures enzyme activity by continuous monitoring of absorbance over fixed time periods at a preset wavelength. Enzyme activity is subsequently calculated using the molar absorptivity of the substance measured. It is therefore essential that a check of the absorbance accuracy of the instrument can be made independently of reaction rate measurement.

The standard output device of the analyser is a $100 \mathrm{mV}$ full-scale deflection potentiometric recorder which is equivalent to a maximum absorbance change of $0.2 \mathrm{~A}$.

The operating sequence of the analyser for each sample is as follows:

(1) The initial absorbance of sample and reaction mixture is measured and recorded, and the voltage equivalent to that absorbance is offset to provide a zero pen position on the recorder.

(2) The subsequent absorbance change is then continuously monitored for a preset time period.

The auto zero device allows a calibration check on the full scale of the instrument response, but the output mechanism prevents conventional assessment of the photometric accuracy of the instrument (Rand, 1960).

The existing method provided by the manufacturer covers only a very limited absorbance range between about 0.4 and 0.6 A (LKB 8600 Instruction Manual) and a need therefore exists for one which will encompass as wide a scale as possible.

\section{Material}

An aqueous solution of naphthol green (BDH microscope stain grade) was prepared, filtered, and diluted in glass-distilled water to give an absorbance of about $2 \mathrm{~A}$.

Twenty-five serial dilutions were then prepared to cover the absorbance range $0.09 \mathrm{~A}$ to $2.235 \mathrm{~A}$. It is necessary that the difference between any two consecutive solutions be less than the full-scale deflection of the recorder, ie, $\mathbf{0 \cdot 2} \mathrm{A}$. Naphthol green has a flat absorbance spectrum in the region of $\mathbf{3 4 0}$ $\mathrm{nm}$, thus minimizing any variation due to wavelength error.
Method

The cumulative and differential absorbances of 17 solutions of naphthol green were measured at 340 nm on a precalibrated (Rand, 1960), double-beam, grating spectrophotometer (Perkin-Elmer 124). Absolute absorbance measurements were made by reference to distilled water.

Differential absorbance between consecutive solutions was measured by placing each sample in the 'test' compartment with the preceding sample in the reference compartment. The cumulative and differential absorbances are then shown (table).

\begin{tabular}{|c|c|c|c|c|}
\hline \multirow{2}{*}{$\begin{array}{l}\text { Naphthol } \\
\text { Green } \\
\text { Solution }\end{array}$} & \multicolumn{2}{|c|}{$\begin{array}{l}\text { Perkin Elmer } 124 \\
\text { Absorbance at } 340 \mathrm{~nm}\end{array}$} & \multicolumn{2}{|c|}{$\begin{array}{l}L K B 8600 \\
\text { Absorbance at } 340 \mathrm{~nm}\end{array}$} \\
\hline & Cumulative & Differential & Cumulative & Differential \\
\hline 1 & 0.090 & & 0.094 & \\
\hline 2 & 0.175 & 0.085 & 0.174 & 0.080 \\
\hline 3 & 0.270 & 0.095 & 0.268 & 0.094 \\
\hline 4 & 0.365 & 0.095 & 0.360 & 0.092 \\
\hline 5 & 0.450 & 0.085 & 0.434 & 0.074 \\
\hline 6 & 0.540 & 0.090 & 0.526 & 0.092 \\
\hline 7 & 0.625 & 0.085 & 0.611 & 0.085 \\
\hline 8 & 0.710 & 0.085 & 0.703 & 0.092 \\
\hline & & 0.095 & & 0.080 \\
\hline 9 & 0.805 & 0.090 & 0.783 & 0.089 \\
\hline 10 & 0.895 & 0.085 & 0.872 & 0.089 \\
\hline 11 & 0.980 & & 0.961 & \\
\hline 12 & 1.065 & 0.000 & 1.050 & 003 \\
\hline 13 & $1 \cdot 160$ & 0.095 & $1 \cdot 142$ & 0.092 \\
\hline 14 & $1 \cdot 245$ & 0.085 & $1 \cdot 230$ & 0.088 \\
\hline 15 & 1.335 & 0.090 & $1 \cdot 310$ & 0.080 \\
\hline & & 0.085 & & 0.088 \\
\hline 16 & 1.420 & 0.090 & 1.398 & 0.082 \\
\hline 17 & 1.510 & & 1.480 & \\
\hline
\end{tabular}

Table Cumulative and differential absorbances of solutions of naphthol green measured at $340 \mathrm{~nm}$

The absorbance of those solutions were made on the LKB 8600 analyser using the following protocol:

1 Set the instrument and recorder to zero as laid $\stackrel{?}{+}$ out in the instruction manual.

2 Set reaction course to 'increase' and absorbance range of $0-0.2 \mathrm{~A}$.

3 Prepare cuvettes as follows:

To first cuvette add $1.0 \mathrm{ml}$ glass-distilled water.

To each subsequent cuvette add $1.0 \mathrm{mil}$ of each of 


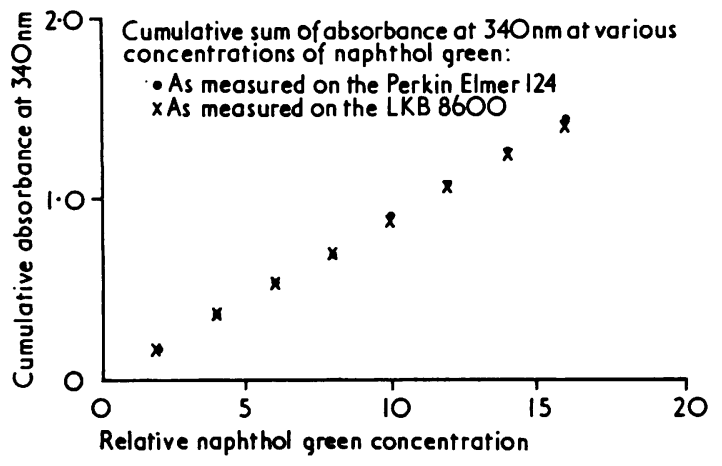

Figure Cumulative sum of absorbance at $340 \mathrm{~nm}$ at various concentrations of naphthol green: as measured on the Perkin Elmer 124; $\times$ as measured on the LKB 8600

the naphthol green solutions in ascending order of concentration.

4 Place the racks in the input magazine and move cuvette 1 to the measuring position.

5 Switch to 'set light' with the background slider in position 0.0 and bring the recorder pen to the zero position with the appropriate light control.

6 Press the 'feed' control to move the next cuvette into the measuring position and note the recorder pen deflection.

7 Reset the recorder pen to zero with the 'light' control.

8 Repeat stages 6 and 7 until all cuvettes have passed the measuring position.

9 Calculate the absorbance difference between consecutive solutions by simple proportion given that full-scale deflection is equivalent to $0.2 \mathrm{~A}$.

10 Compare the cumulative and differential absorbances with the reference values obtained from the precalibrated, accurate spectrophotometer.

\section{Results}

The differential and cumulated absorbance values for the LKB 8600 analyser and the reference spectrophotometer are given in the table. The cumulative absorbance values for both instruments are plotted in the figure.

\section{Discussion}

The photometric accuracy and therefore linearity of enzyme reaction rate analysers, throughout their entire absorbance range, are critical to the accuracy of the enzyme assay, because of the calculation which involves molar absorptivity (usually of NADH).

The results presented demonstrate that at $340 \mathrm{~nm}$ the absorbance accuracy of our LKB 8600 analyser is not significantly different from that of our reference spectrophotometer. The presentation of results as differential absorbance is particularly appropriate since enzyme activity measurement occurs over a maximum absorbance change of $0.2 \mathrm{~A}$, although the initial absolute absorbance levels vary with both the assay system involved and the absorbance of the diluted sample.

\section{Conclusion}

This method represents a simple and rapid technique for assessing photometric accuracy and linearity and is equally applicable with, for example, p-nitrophenol at $405 \mathrm{~nm}$ or indeed any suitable solutions or neutral density filters. It is a procedure which might easily be adopted as a periodic exercise to be incorporated into the routine quality control programme of any laboratory which possesses this analyser.

\section{References}

Rand, R. N. (1960). Practical spectrophotometric standards. Clin. Chem., 15, 839-863.

LKB 8600 Instruction Manual, 45-47. 